\title{
DEFAULT HANDLING IN INCREMENTAL GENERATION
}

\author{
Karin Harbusch†, Gen-ichiro Kikuit, Anne Kilger † \\ ‡ ATR*, Fax: (+81 7749) 5 1308, E-mail: kikui@itl.atr.co.jp \\ † DFKI, Fax: (+49 681) 302 5341, E-mail: harbusch|kilger@dfli.uni-sb.de
}

\begin{abstract}
Natural language generation must work with insufficient input. Underspecifications can be caused by shortcomings of the component. providing the input or by the preliminary state of incrementally given input. The paper aims to escape from such dead-end situations by making assumptions. We discuss global aspects of default handling. Two problem classes for defaults in the incremental syntactic generator VM-CIEN are presented to substantiate our discussion.
\end{abstract}

\section{MOTIVATION}

Natural Language Generation, i.e., the process of building an adequate ntterance for some given content, is by nature a decision-making problem (Appelt, 1985). Intemal decisions are made on the basis of the specified input. Unfortunately, input information can be insufficient in two respects:

- If the input structure for generation is provided by another AI-system, global problems in producing sufficient input information for the generator may occur, e.g., because of translation mismatches in machine translation (Kameyama, 1991). In this case, the generator either has to use a default, or lormulate a request for clarification in order to be alsle to continue its processing, i.e., to produce an utterance. During simultaneous interperation requests are rather unusual. Here defaults allow for a standalone landling of the problem. For example, problems during speech recognition of antomatic interpretiation can lead to results like "the (man/men) will come to the hotel tomorrow". If the system is not able to give a preference for one of the alternatives, e.g., by cvaluating context information, the gencrator has to choose

"The author is currently at N'TT' Nelwork Information Systems Laboratories (kikui(inttuly.ntt.jp). a probable number value on its own to complete verbalization.

- Furthermore, for incremental generation, the input information is produced and handed over step by step, so that it can be temporarily incomplete - although as a whole it may become sufficient. This behaviour of a generator is motivated by psycholinguistic observations which show that people start speaking before all necessary linguistic material has been chosen (e.g., articulating a noun phrase before the dominating verb is selected). As a consequence of underspecification, incrementall generation is essentially based on working with defaults. Elements are uttered before the processing or input consumption has been finished. (Kitano, 1990) gives an example for defaults in the context of simultaneous interpretation: In Japanese, negation is specified at the end of the sentence white in English, it las to be specified in front of the funite verb. 'Therelore, during Japanese-English translation, where analysis, transfer, and generation are performed in a parallel and incremental way, the system has to commit, e.g., positive value hefore knowing the actual polarity'.

Gencrally speaking, defaull handling specifies how processing, i.e., further decision-making, can continuo withont, sulficicnt, input information. So, onc can compare default handling with advice to the system. For reasons of uncertainty of assumptions, incremental systems with this facility must be able to repair the default decision when the assumption turns out to be wrong by information given later. Catching on to the above example, there can be a negation specifier given at the end of the Japancse juput sentence which cannot be simply integrated into the output

\footnotetext{
${ }^{1}$ Alternatively, tile system coukl use the dialogue context to infer a megalion value $+/-$.
} 
sentence because the finite verb has alrearly been uttcred. In this case, the output has to be repaired, e.g., by repeating parts of the utterance: "I will be able to meet you ...oops ... I won't be able to meet you at the hotel this evening."

In the following sections, we argue for the appropriateness of processing-conforming default handling. Basically, the processing-conforming mode makes the overall system homogeneous because the combination of default-caused processing and input-licensed processing requires no specific description. The homogeneity becomes especially helpful in the case where the input, verifies the default assumption rendering unnecessary any recomputation. For the opposite case where the defanlt must be withdrawn we lave to mark all defanlts. Fven more homogeneity is introduced to an incremental system if the default descriptions are given in terms of input specificalions. This representation allows for easy checking the coincidence between a chosen default and input given later.

The content of this paper can be summatrized as follows. Section 2 provides a general description for defaults in generation emplatsizing the specific requirements in an incremental system. After identifying the conditions under which defaults are triggered (section 2.1), the application of a default (section 2.2) and the defintion of its description (section 2.3) is outlined. The crucial calse of removing defanlts not coimciding wilh newly arriving input in an incremental system is discussed in section 2.4.

In section 3, this mechanism is appliexl to the incremental sentence generator VM (IEN. In the beginning of the section, the basic design of the system is ontlined. Later on, default handling is included and exemplified for two general cases.

In the final section we summarize the main results of the paper. linthemore, we discuss how default handling can be adingted to multilingual generation, as recuired by the: spech-to-speech translation system VERRBMOBII. (Block et al., 1992).

\section{GENERAL DISCUSSION OF DEFAULTS}

In the literature of non-incremental generation, the need for defaults is hardly ever taken into account. The common point of view restricts the input to be sufficient for generation (see, e.g., the Text Structure by (Metecr, 1990) for a syntactic generator). In incremental generation, most authors agree on the necessity of using clefaults (see, e.g., (I) Smedt, 1990; Kitano, 1990; Ward, 1991)). Nevertheless, they do not in sufficiont depth answer the question of how to guicle the processes of default handling and repair within a generator. 'This problem is the starting-point for the following considerations.

Wo assume that generation is a decision-making process with the aim of producing a plausible utterance based on given information. As mentioned in section 1 , there are cases where this process stops (cansed by underspecificalion of the input) before finishing its output.

We define a moclule named default handler which tries to resume the process by giving advice to it, i.e., by making assumptions about the missing input specification. With respect to this task it is cliscussed

1. in which situations defanlts are applied (see section 2.1),

2. how default landling is integrated into a system (see soction 2.2),

3. how the knowlerge for defanlt landling is described (see section 2.3), and

1. how assumptions are cancelled when blicy tum out, to be inconsistent, with newly arriving input (see section 2.4).

In incremental generation, as mentioned in section 1, interleaved iuput consumption and ontput production canses specific defanlt sitnations. An incremental processing scheme inllows for an increase of effeciency and flexibility, e.g., by making the analysis and gencration processes of a system for simultaneons interpretation overlap in time. There are two competing goals of incremental generation for spoken output, that must be taken 
into account when estimating the usefulness of defaults:

Fluency: Long hesitations should be avoided during the production of an utterance, in order to be acceptable to the hearer ${ }^{2}$.

Reliability: Errors in an utterance may cause misunderstanding. In most cases, errors should be recovered by appropriate selfcorrections $^{3}$. Excessive use of self-corrections or erroneous expressions should be avoided because they decrease intelligilbility of the utterance.

Obviously there is a trade-off between fluency and reliability: maximal reliability requires 'secure' decisions and therefore leacls to ontput delay. On the other hand, maximal fluency necessitates the use of assumptions and repair, respectively.

\subsection{When to Trigger Default Fandling}

We define as default situation the situation where a generation system lias not yet finished the utterance but at the same time has consumed all given input and is not able to continue processing. In non-incremental generation, this corresponds to the fact that the input lacks necessary information, because the entire input is assumed to be given at one time (e.g., the undecidable number value of the example described in section 1). Thus, default handling should be triggered immediately.

In incremental generation, however, the system may get a new piece of information hater. on that enables it to continue processing (e.g., the specification of a negation value + as outlined in the example in section 1). 'Tlurefore, possible alternatives are cither to wait for the next input or to trigger defanlt handling. The former violates the fluency goal, the latter may violate the reliability goal. We propose the explicit use of time-limits for drlay intervals ${ }^{4}$.

\footnotetext{
"IIumans often fill such pauses with fillers like "er" or "what shall I say".

"Sometimes, correction is unnecessary if (like speaker believes that) the hearer can infer the inlended utterance from erroneous speech.

${ }^{4} \mathrm{An}$ explicit parameter expressing the desired degree of fluency influences the time--linits.
}

Furthermore, the certainty of a default is described by a value. As soon as a default situation is identified, the certainty of the default is checked to sec whether it exceeds a predefined threshold that determines the degree of fluency/reliability ${ }^{5}$.

Each application of a default decreases the global certainty of the system's state. Consequently, there shonld be a limit for lhe maximal number of defaulls applicable to the same sentence.

\subsection{How to Integrate Default Handling}

Basically, there are two strategies to integrate default landling into ongoing processing.

Defaults may be haudled in a way that differs from the 'normal' processing of the system, e.g., as short-cuts. One advantage can be an efficient landling of defaults. Furthermore, the designer of the default component is completely free in deciding abont the realization of defaults in the system. $A$ disarlvantage is the difficulty of providing consistency between default cansed and inputlicensed processing.

Alternatively, the ongoing processing can deal with the default values in an ordinary manner (processing conforming defanlt handling). This may be less efficient but guarantrees consistency during processing, especially in case of a replacement by an input-licensed value. For incremental generation, the system las to provide repair facilities in any case. So, they can also be used for non-monotonic: modificalions of defunld caused results. We take this option in order to make the overall system homogrmoous.

\subsection{How to Describe Defaults}

The knowledge source that is used for default handling should provide the most plansible actions for a default situation. We represent the knowledge as a set of heuristic rules called defaull descriptions. A default description defines a set of operations that should be carried

\footnotetext{
"The basis for assigning certainty values to defuults should be a corpus sludy that can be used to find statistical evidence for various features with altemalive values (like mumber, voice, ..., see, e.g., (Bock and Warren, 1985)).
} 
out in a certain situation where the generation process can not be continued. A default description has the following form:

$$
\left[\begin{array}{l}
\text { default } \\
\text { preconditions }
\end{array} \Rightarrow \begin{array}{l}
\text { default } \\
\text { bodly }
\end{array} ; \begin{array}{l}
\text { cerlainty } \\
\text { value }
\end{array}\right]
$$

The set of defaull preconditions defines tests that are applied to the given situation in older to find out whether the corresponding default body can be activated. They include tests for the existence of particular information, tests for the structure under creation and tests for the state of processing.

The default body describes how to continue processing with defaults in an adeculate way. For incremental systems, we propose to express the body as a specification of input increments. An important prerequisite is that the size of increments is defined flexibly enough to cope with varying amounts of information. Obviously, an important advantage of this approach is homogeneity of the overall system. Especially, the lomogeneons representation of default-caused and input-licensed structures is the casiest and most direct way to test concidences or contradictions: between defanlt-specified and input-cansed values. In section 3, this approach is outlined by different examples. For non-incremental systems, an operational approach is preferable since there is no way to consume additional input increments, presupposing that, the input has been considered as a whole before a default situation occurs ${ }^{6}$.

If several default preconditions are anplipcable, the certainty values for delault descriptions are examined to find which provides the system with the most plansible axtion.

The individual defunlt descriptions should take into account the global constraints for processing stated in the knowlerge sonrces of the system. For example, the assumption of nominative case for a German NI complement can regularly be made only once for the

\footnotetext{
${ }^{6}$ The difference between incremental and nonincremental generation becomes smaller, if we assume that defaults in a non-incremental system can loe triggered after the system has only considered parts of its input information. In this case, the input considered after default, handling becomes comparable to later increments.
}

same verb. For reasons of lomogeneity, the default description should at least be compatible with the specifications of the knowledge used for hasic processing. In order to guarantee consistency, default descriptions should merely contain what is orthogonal to the basic knowledge sources.

\subsection{How to Cancel Defaults}

The repair of false assumptions is a crucial point of default landling in the context of incremental processing because the default information does not remain locally but can cause further decisions of the system. Contratily, for non-incremental input there will be no value given that can contradict default vallues.

$\Lambda$ s a first step of repair, inconsistencies between input,-provided and defanlt--cansed valtees are identified by simply matcling the values. Then eflects of the respective defaults are withdrawn introducing the input--provided values into the system. Generally, a decision during generation influences other decisions all over the system. 'Thus the eflect of a definlt body may be propagated through the entite systcm (e.g., choosing a construction of main clatuse with cansal subordinate clanse influences the choice of syntactic realizationss).

Roughly speaking, withdrawing a defanlt assumption can be realized by backtracking to the earlier state of the system where the default had been introduced or by non-monolomir changes to the current state of the system. 'T'he disadvantage of backtracking is that, partial results are thrown away which could be rensed during further processing. Nonmonotonic changes preserve these results. In this frimework, cancelling defunlts requires the system to identify which resultis are caused by defanlt handling. Dependency links be1.ween the immediate result of a default body and results of the influenced decisions allow for this identification. The disadvantage of non-monotonic changes is the complexity of computation, e.g., supported by a truth maintenance system. When designing an incremental system, simple backtracking is ruled 
out because the part of the sentence uttered cannot be withdrawn after it has been perceived by the addressee of the message ${ }^{7}$.

So, we end up with a processing-conforming default handler for generation realizing repair by non-monotonic changes.

\section{EXAMPLES OF DEFAULTS IN VM-GEN}

The adaptation of our general discussion of default handling to the system VM-GEN not only provides concrete examples for the rearer but also shows that a homogencous combination of default handling, regular processing, and utterance repair is possible.

The syntactic generator VM-GEN is a lurther development of TAG-GEN (Kilgar, 1994) within the framework of VERBMOBIL, a speech-to-speech translation system. It.s usefulness for simultaneons interpretation results from its incremental and parallal slyle of processing. VM-GEN is able to consume input increments of varying size. These incrementis describe lexical items or semantic relations between them. Single input increments are handed over to objects of a distributed parallel system, each of which tries to verbalize the structure that results from the corresponding input increment. VM-GEN uses an extension of Tree Adjoining Grammers (TAGs, of. (Joshi, 1985)) as its syntactic representation formalism that is not only adequate for the description of natural language but also supports incremental generation (Kilger and Finkler, 1994).

In the following, we introduce examples for default processing triggered during the Cierman inflection process in VM-GEN to sulstantiate the global statements made in section 2 . Inflection uses some syntactic properties of an element to compute its morphological form. This information has partly to be specified in the input (e.g., the number for a noun) and is partly inherited from other el-

\footnotetext{
${ }^{7}$ If some phrases influenced by defaulls lave already been verbalized, the effect of verbalization can be cancelled by using repair words like "oops" or "solry" when starting the modified utterance.
}

ements (e.g., the number for a verb or the case for a noun). The two reasons for missing information necessitate different methods of treatment which nevertheless both can unjformly be integrated into regular processing.

If information of the first type is missing (e.g., because of problems during analysis, see section 1), an assumption can be made locally by simulating the respective part of the input. The default for missing number information in VM-GEN would look as follows:

$\left[\begin{array}{l}(\text { cat }(O B J)=N) \\ (\text { number }(O B J)=N I L)\end{array} \Rightarrow \begin{array}{r}(\text { ENTITY OBJ } \\ (\text { number } s g))\end{array} ; 0.8\right]^{8}$

The set of default preconditions is applied to all ${ }^{5}$ objects (OB.I) of VM-GEN in order to test the kind of underspecification ('number' in the example). 'The delault body introduces a new value (sg) by creating an input increment as a default. The test for coincidence with the input licensed value is realized by a comparison in the objects of VM-GEN. There is a mique association of input increments and objects of VM-GEN (OBJ is used as identifier) that allows for translating an input modification into a molification of the state of the respective object. In case of contradictions the defanlt and all default-cansed decisions are revised ${ }^{10}$ (see below).

Maling an assumption caul be influenced by globul constraints. An cxample, which is well studied in psycholinguistics, is the utterance of a noun before the verb has been chosen. If, e.g., the noun "Besucher" (English: "visitor") is known to be the agent of an action, it may be uttereel as sulb ject in the first position of the sentence by default. This treatment presupposes the choice of a 'dummy' verb, which at least subcalegorizes a subject and has active voice ${ }^{11}$. The use of a dummy

\footnotetext{
"liN'TITY' introlnces information about a lexical item. For reasons of incrementality, there may be several ENTI'TY-packages specified for the same item whith are composed to receive the global information. for certainly values, we use values between 0 and 1 , where 1 means high reliability.

"In the achual implementation we preselect candidales with missing values for reasons of efficiency.

${ }^{16}$ for ongoing work on repair in VM-CiEN see (Finkler, 1991).

${ }^{11}$ This kind of expansion is called "provisional upward expansion" by (De Simedh, 1990).
} 
verb and an underspecified verbal structure the NP is integrated into allows for a simple global test that rules out the same case value assignment to different. NP complements as it is required for most of the German verbs. This rule is represented in the grammar as a part of the description of subcategorization frames for verbs. For reasons of homogencity we use the information stored in the syntactic knowledge sources of VM GEN for expressing syntactic constraints during default handling as well. 'The advantage of this' approach is, that processing is continued in a consistent way, which eases the introduction of the input-licensed value. One default for choosing a missing case-value is specified as follows:

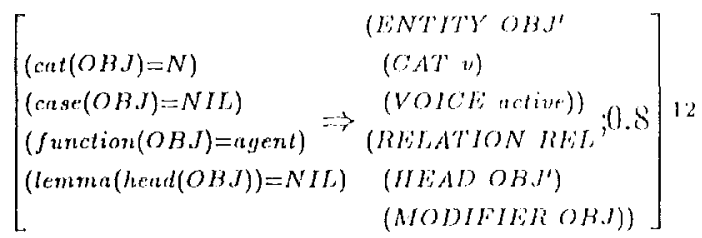

The default preconditions of the rule characterize a situation where an object (O) 3,J) contains no information about the case but identifies the input category as ' $N$ ' for noun. Finthermore, the semantic function of the object is specified as 'agent:' but no verb defined yot. (lemma(head $(O B J))=$ NIL) in the liead object. 'That is why, the $\mathrm{N}$-object cannot inlerit. a case value and also does not know whether it is allowed to occupy the front position in the utterance.

Evaluating the default boty, the system creates a V-object OB.J'. On the basis of the input, information in (ENTITYY OR3.J' ...) it. chooses a minimal syntactic structure from the inheritance net of the grammar, that just desribes a verb category without concrete filler (a dummy verb) plus a subject complement and active voice for the verbal phrase. Now, the $\mathrm{N}$-structure is combined with the Vistructure of the introduced V-object as cluring normal processing. Therefore, the case value can be inherited. Additionally, the first position

\footnotetext{
12'RELATION' introduces the specification of a rolation between two lexical items which are identified by the names of their objects.
}

can be assigned to the subject which can be nititered now.

The basic VM-GEN module provides repair strategies in order to allow for the specification of additions, modifications and deletions of input increments, i.c., to model a flexible input, interface. Three features of the system are basically used for repair: First, input increments are unichely associated with objects of VM-GWN, so that input modifications can be translated into modifications of the objects' states. Second, each modification of an object's state makes it compare new and old information. In case of a difference, the modified parts are sent to all concerned objects. Third, the dependency relations that determine the communication links between oljects allow for a hicrarchical organization of the objects, which is the hasis for syuchronizing repaitr.

A repair must be triggered in the exam ple described above if, e.g., a verb wilh voice passive is actually specified. In this case, the mapping of the semantic role 'agent' to the syutactic: linction 'subject' is revised. 'T'he agent now has to be realized as part of a "von"-phrase, eg. "dieser Termin wird yon dem Besucher gewïnschte." (word-for-word: "this date is whished by the visitor (dative objoct)"). Furthermore, the olject checks whether the previously uttered part of the sentence includes some of the revised material (i.e, whether the olject itself lias participatol in uthering). If this is the casce, it sends an crore message up to the uppermost object of the licearchy that actually is engaged in nutering. This object is alse to synchronize global repair. Up to now, we just realized a simple reparir strategy that consists of repeating the concened parts of the utterance, e.g. "der Besucher ... äl ... dieser Temin wird non. dem Brsucher gewünselit".

\section{DISCUSSION}

This paper proposes a processing-conforming defant handler for generation realizing repair by non- monotonic changes. We provide the system with default descriptions. The set of default preconditions expresses possible rea- 
sons for dead end situations. A default is triggered, if the preconditions match the current situation and the certainty value of the default exceeds the predefined threshold. The default body is expressed in terms of the missing input specification in order to make the system work homogeneously. We have verified the advantages of processing-conforming default handling by implementing a default. handler for VM-GEN.

As future work, we will extend the default preconditions towards handling complex contextual information. We will apply default handling to microplanning and lexical choice within VERBMOBII. With respect to a sophisticated output, we aim to combine VMGEN with a flexible repair component.

The system VM-GEN is used in the VERRBMOBIL scenario for multilingual generation (English, German, and Japanese). We mean by multilinguality that the same processing is applied for different, languages. In the underlying knowledge sources language-specific constraints are defined. Default handling can be easily adapted to the requirements of multilingual generation by using language specilic default-descriptions.

For all knowledge sources the question arises how knowledge can be shared. We intend to use core knowledyr sources for representing common phenomena. The core set of default descriptions for English and Gorman, e.g., contains the description of a reaction to a missing number value for a noun. We aim to develop an efficient storing mechanism using a hierarchy of locally intersecting core tescriptions.

\section{References}

Appelt, D. (1985). Planning English Sentenecs. Cambriclge, MA: Cambriclge University Press.

Block, H.-U., Bosch, P., Engelkamp, J., v. Mahn, W., Hauenschild, C., Höge, H., Rohrer, C., Tillmann, II., G., Wahlster, W., Waibel, A. (1992). Wissenschaftliche Ziele und Notzpläne für das VERBMOBI,-Projekt. Technical report, German Research Conter for Artificial Intelligence (DFKI (imbH), SaIIbrïcken, Germany, 1922.
Bock, J., and Warren, R. (1985). Conceptual accessibility and syntactic structure in sentence formulation. Coynilion, 21, 47-67.

De Smedt, K. (1990). Incremental Sentence Gencration: a Computer Model of Grammatical Encoding. PhD thesis, Nijmegen Institute for Cognition Rescarch and Information Technology, Nijmegen, NICI TR No 90-01.

Fin-

kler, W. (1994). Performing Self-Corrections During Incremental Natural Language Generation. Document, German Research Center for Artificial Intelligence (DFKI GmbH), Saarbriicken, Germany, 1994. to appear.

Joshi, A. (1985). An Introduction to TAGs. Technical Report MS-CIS-86-64, LINC-LAB31, Department of Computer and Information Science, Moore School, University of Pennsylvania.

Kancyama, M., Ochitani, R., and Peters, S. (1991). Resolving Trmaslation Mismatches Wilh Fuformation Flow. 29th Annual Mecting of the Association for Computational Linguistics, Berkeley, CA, pp. 193-200.

Kilger, A. and linkler, W. (1994). TAG-lused meremental Generution. Technical report, German Research Center lor Artificial Intelligence (DEKI CimbH), Saarbriicken, Germany. to appear.

Kilger, A. (1994). Using UTAGs for Incremental and Parallel Generation. Computational Intelligence. to appeas.

Kitano, Il. (1990). Ineremental Sentence Production wilh a Parallel Marker-Passing Algorillm. 13th International Conference on Computational Linguistics, IIelsinki, Finland, p). $217-222$.

Mnteer, M. (1990). The "Gencrution Giap": the Problem of Expressibility in That Plan. ning. Department of Computer and Information Science, University of Massachusetts, Amlierst, MA, BBN Report No. 7347.

Ward, N. (1991). A Flexible, Parallel Morlel of Nalural Langutage Generation. PhD thesis, Computer Science Division (ELCS), Universily of California, Berkoley, CA, Report No. UCB/CSD $91 /$ ( 229 . 